\title{
A Protocol for Evaluating the Accuracy of 3D Body Scanners - Landmark Locations and Surface Shape
}

\author{
Makiko KOUCHI ${ }^{{ }^{a}}$, Masaaki MOCHIMARU ${ }^{a}$, Bruce BRADTMILLER ${ }^{b}$, Hein DAANEN ${ }^{c}$, Peng Li ${ }^{d}$, \\ Beatriz NACHER ${ }^{e}$, Yunja NAM ${ }^{\dagger}$ \\ ${ }^{a}$ Digital Human Research Center, AIST, Tokyo, Japan; ${ }^{b}$ Anthrotec Inc., Yellow Springs (OH), USA; \\ ${ }^{\mathrm{C}} \mathrm{TNO}$, Soesterberg, The Netherlands; 'US Army, Natick (MA), USA; \\ ${ }^{\mathrm{e}} \mathrm{IBV}$, Valencia, Spain; 'Seoul National University, Seoul, Korea
}

\begin{abstract}
We propose a protocol for evaluating the accuracy of scan-derived surface shape and the repeatability of scan-derived landmark locations. Following existing Japanese and German domestic standards, we propose to use a calibrated artefact (e.g. a sphere about $120 \mathrm{~mm}$ in diameter) for evaluating the accuracy of surface shape. To evaluate the repeatability of landmark locations, we propose to use an anthropomorphic dummy with premarked landmark locations. The test objects are measured by the 3D body scanner to be evaluated, and evaluation parameters are calculated from the measured data. A round-robin test was conducted in six different institutes using 19 body/head/foot scanners produced by 10 companies/institutes. The purposes of the round-robin test were to evaluate whether the test objects could be scanned by different body scanners, to evaluate various scan locations for the test objects within the scanning volume, and to examine the quality parameters to be reported. As a result, the proposed test objects could be measured and the data exported by all systems except one, which did not export the ball measurement. Quality parameters are useful for quantifying the accuracy of scan-derived surface shape and repeatability of landmark locations, and providing a basis for the agreement between anthropometric data providers and data users. Since required accuracy depends on applications, it is important to examine results from different scan locations to evaluate the quality of scan-derived measurements rather than considering only the worst case.
\end{abstract}

Keywords: scan-derived measurement, landmark location, repeatability, accuracy, evaluation protocol

\section{Introduction}

A body scanner can obtain three types of measurements, body dimensions, landmark locations, and surface shape. Since these measurements are not traceable to the international standard of the length, their accuracy needs to be evaluated to provide the basis for the agreement between data providers and data users. ISO 20685 [1] establishes a protocol for evaluating the comparability between scan-derived 1-D measurements and those obtained by traditional methods. Landmark locations and body shape have recently seen increasing utilization through homologous body shape modeling $[2,3]$. ISO TC159/SC3/WG1 has been working to develop international standards related to the quality control of anthropometric data. Considering the present situation, ISO TC159/SC3/WG1 plans to create an international standard that establishes a protocol for evaluating the accuracy of 3D shape measurements and repeatability of landmark locations.

There are Japanese and German domestic standards that establish methods of acceptance tests and reverification of optical 3D measuring systems. These standards use balls, ball-bars, and rectangular parallelepipeds as test objects to evaluate the accuracy of scanner systems [4-7]. International standardization of this protocol is in progress by ISO/TC122.

In a tentative protocol for evaluating body scanners by ISO TC159/SC3/WG1, two types of test objects are used. A ball is used to evaluate the accuracy of the surface shape following the above-mentioned standards, and a dummy is used to evaluate the repeatability of landmark locations. To validate this tentative protocol, we conducted a round-robin test using these test objects. The purposes were to evaluate the ability of different scanners to scan and export data from the test objects, and to obtain materials for discussions on the locations of measurements for test objects within the scanning volume and quality parameters to represent the scanner performance [8]. Since then we evaluated the protocol using two more scanner systems, and developed software to calculate quality parameters of landmark locations. In this paper, we present our tentative protocol and results of the round robin test, and propose quality parameters to quantify the quality of the shape measurements repeatability of landmark locations. 


\section{Artefacts}

\subsection{Ball}

In the present protocol, a ball with a diameter of $120.0159 \mathrm{~mm}$ (sphericity $=22.1 \mu \mathrm{m}$ ) was used as one of the test objects. The artefact was a hollow steel ball, surface blasted and treated with TiN to diffuse reflection (Figure 1, left). This treatment was the most successful in a round-robin test conducted in Japan in which balls with several different surface treatments were measured using different 3D measuring systems [9]. The ball was calibrated in National Metrology Institute of Japan, National Institute of Advanced Industrial Science and Technology (AIST), using a coordinate measuring machine (CMM). By using this test object, measurement by a 3D scanner can be traceable to the international standard of the length.

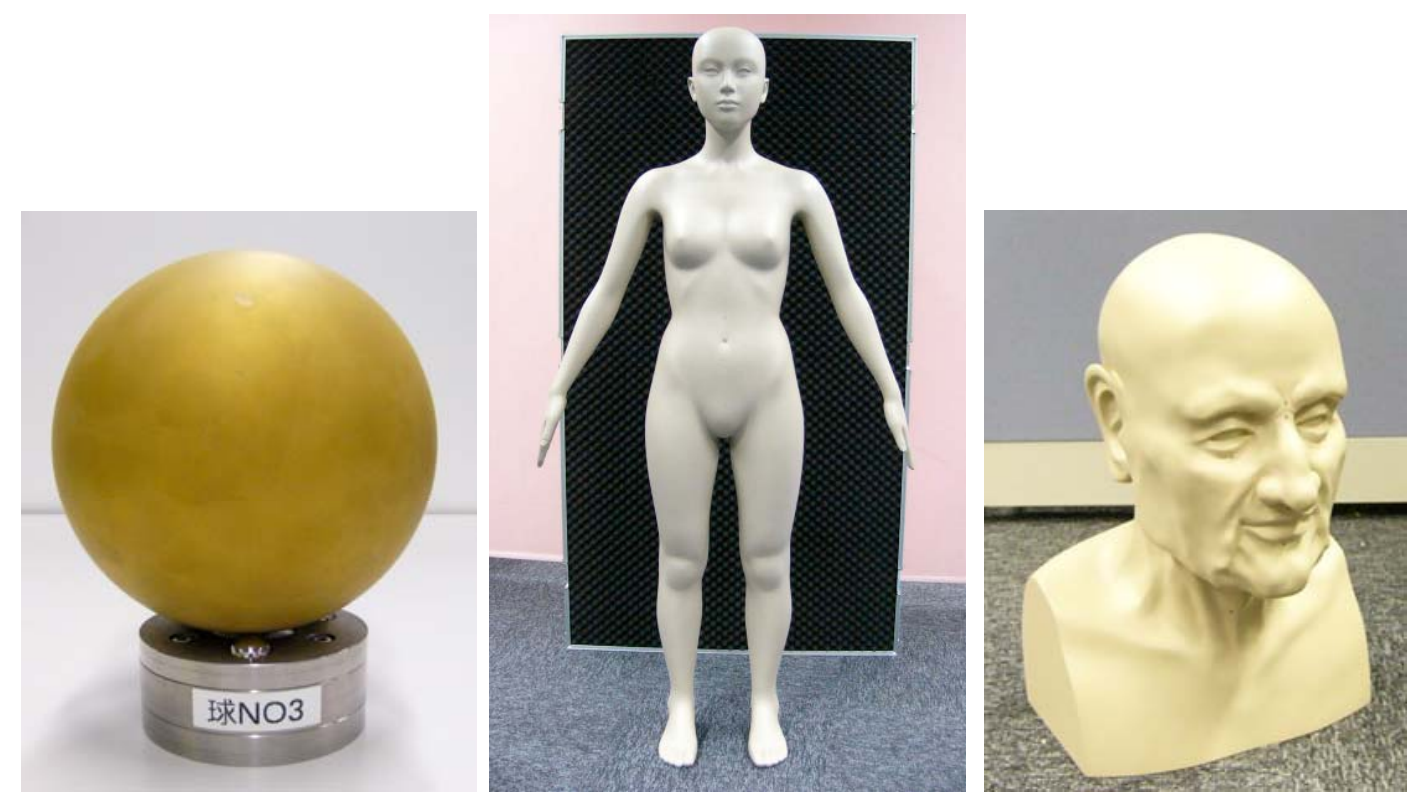

Fig. 1. Artefact. Left: ball ( $\varnothing=120.0159 \mathrm{~mm}$, sphericity=22.1 $\mu \mathrm{m}$ ); Middle: whole body dummy; Right: head dummy.

\subsection{Dummy}

One of the characteristics of body scanners is that they usually accompanied by software to detect marker locations or calculate landmark locations. To evaluate landmark locations, an artefact similar to the actual human body is necessary, because complicated shape of the human body causes occlusion that would not occur in simply shaped objects. We used a whole body dummy with the posture recommended in ISO 20685. This dummy was made of fiber reinforced plastic (FRP) with no movable parts such as joints, and has average body dimensions of Japanese females in their 20s (Figure 1, middle) (Nanasai Co. Ltd., Kyoto). Locations of landmarks are marked with small dents.

For the head dummy, we used a dummy shown in Figure 1, right. The form of the dummy did not reflect the average head form, but was created to have difficult shape features to measure (Nanasai Co. Ltd., Kyoto). Locations of landmarks are marked with small black dots.

We did not use a foot dummy in the present round-robin test.

\section{Measurement of the ball}

\subsection{Measurement locations of the ball}

Measurement locations for the ball were established throughout the volume typically occupied by a human body.

\subsubsection{Whole body scanner}

Nine locations were selected (Figure 2A). In determining these locations, we assumed that the subject stands with arms and legs abducted as recommended in ISO 20685. The accuracy of the ball placement was within $\pm 30 \mathrm{~mm}$ of the target position for vertical, lateral, and antero-posterior directions. 
Placement of the ball is not very accurate, because measurement results are not used for calibration and placement of a ball in an exact position in a space is difficult.

As the ball is very close to a true sphere, measurement of the whole ball is not necessary for the test. However, at least half of the ball should be measured at each location. Therefore, when the scanning volume was too small for locating the ball at the locations specified in Figure 1, the location was adjusted, and the adjustment was reported.

\subsubsection{Head scanner}

Seven locations were selected for measurement (Figure 2B). In determining these locations, we assumed that the scanning volume would range from $300 \times 300 \times 300$ [mm] to $400 \times 400 \times 400$ [mm], and tried to cover the volume. The measurer decides the center of the scanning volume. Location \#1 is the center of the scanning volume. Locations \#2 and \#3 are $80 \mathrm{~mm}$ higher or lower than the center, respectively. Locations \#4 and \#5 are $80 \mathrm{~mm}$ anterior or posterior to the center, respectively. Locations $\# 6$ and \#7 are $80 \mathrm{~mm}$ to the right or to the left, respectively. The accuracy of the ball placement is within $\pm 10 \mathrm{~mm}$ of the target position for height, lateral and antero-posterior directions.

\subsubsection{Foot scanner}

Four locations were selected for measurement (Figure 2C). In determining these locations, we assumed that the size of scanning volume would be about $350 \mathrm{~mm}$ (antero-posterior direction) $\times 150$ $\mathrm{mm}$ (lateral direction) $\times 200 \mathrm{~mm}$ (height). The accuracy of the ball placement is within $\pm 10 \mathrm{~mm}$ of the target position for height, lateral and antero-posterior directions.

$\mathbf{A}$
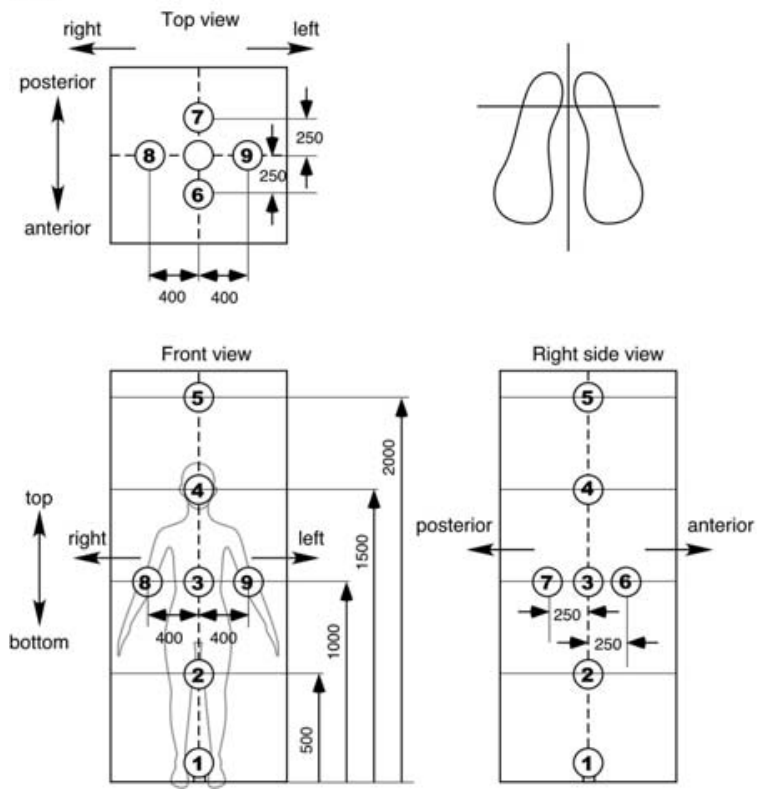

B
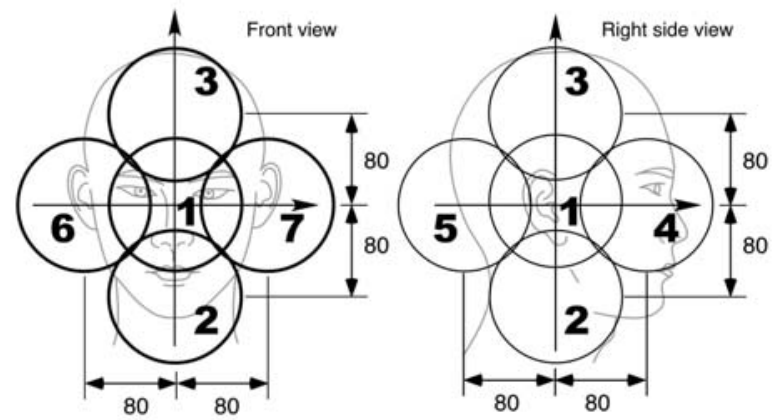

C

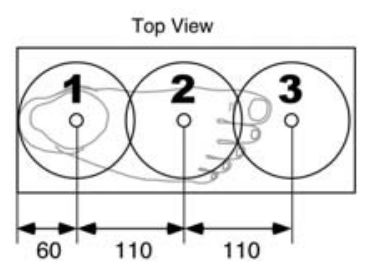

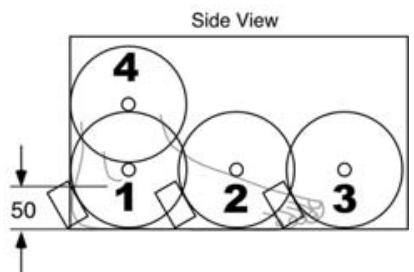

Fig. 2. Locations for measurement of the ball.

A: whole body scanner; B: head scanner; C: foot scanner. (unit: $\mathrm{mm}$ ).

\section{Measurement of the dummy}

\subsection{Landmarks}

\subsubsection{Whole body}

The 47 landmarks shown in Figure 3 were marked on the dummy. Landmarks \#1-\#29 shown by diamonds in Figure 3 are defined in ISO 20685. 


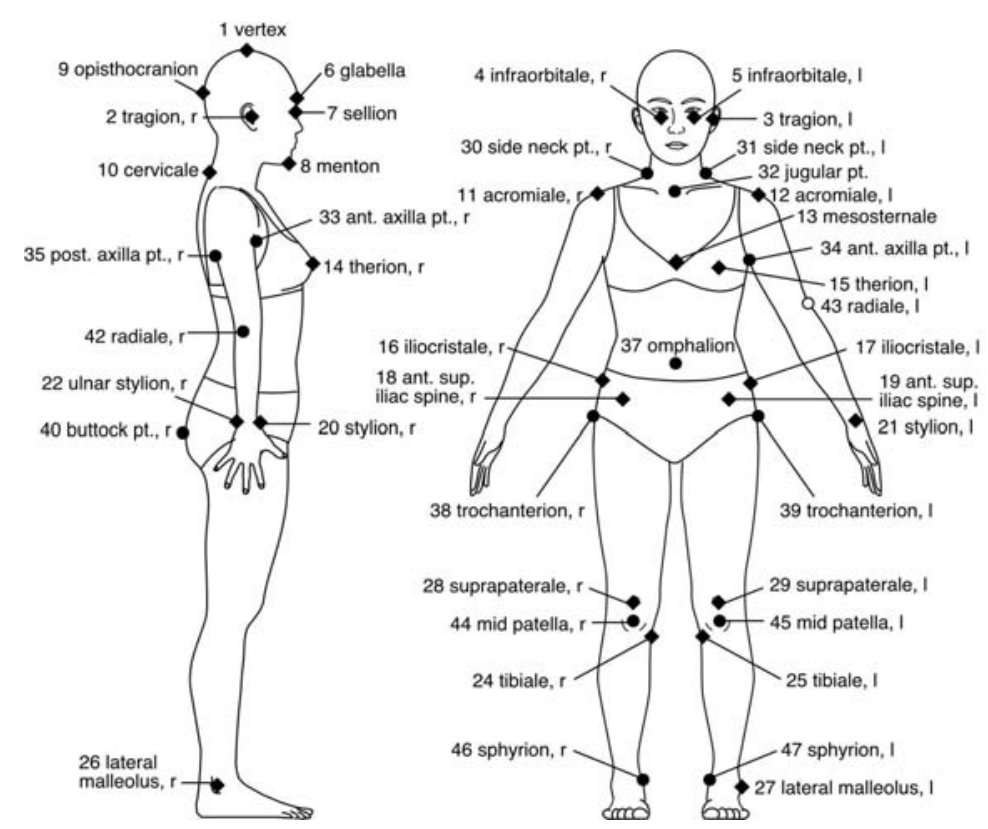

Fig. 3. Landmarks on the whole body dummy. Diamonds indicate 29 landmarks defined in ISO 20685.

23 ulnar stylion, r, 36 post. axilla pt., l, 41 buttock pt., I are not shown.

\subsubsection{Head}

The 14 landmarks shown in Figure 4 were marked on the dummy. Landmarks \#1-\#9 shown by diamonds in Figure 4 are defined in ISO 20685.

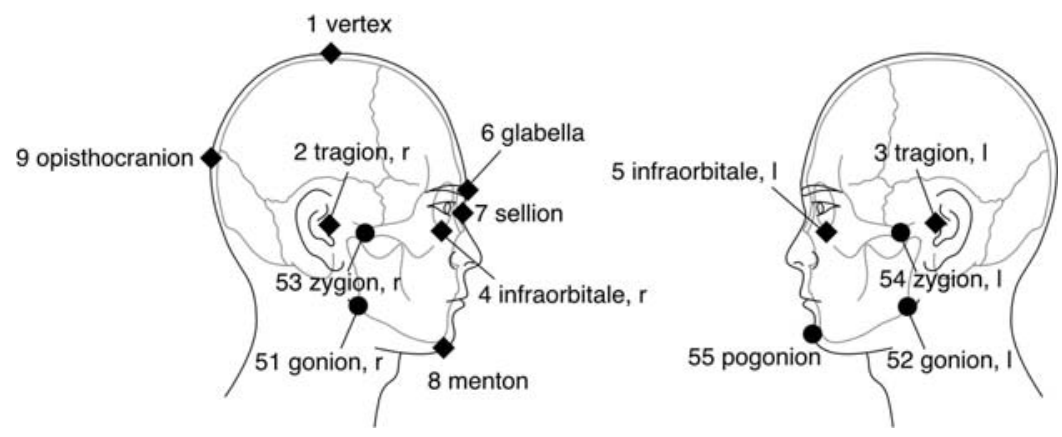

Fig.4. Landmarks on the head dummy. Diamonds indicate 9 landmarks defined in ISO 20685.

\subsection{Measurement}

When marker stickers are used to detect landmark locations, the stickers are attached to the dummy before measurement.

\subsubsection{Whole body scanner}

The dummy is placed at a location where the human subject stands. This location is the base location. The dummy is scanned 10 times. After each scan, location of the dummy is moved slightly to simulate variation in the position of human subjects. Variation in location includes antero-posterior and lateral translations and rotational differences. The following locations are used: 1) the base location; 2) $10 \mathrm{~mm}$ anterior to the base location; 3) $10 \mathrm{~mm}$ posterior to the base location; 4) $10 \mathrm{~mm}$ to the right of base location; 5) $10 \mathrm{~mm}$ to the left of base location; 6) rotated anti-clockwise, with only the right heel placed $10 \mathrm{~mm}$ anterior to the base location; 7) rotated clockwise, with only the left heel placed $10 \mathrm{~mm}$ anterior to the base location; 8) $10 \mathrm{~mm}$ anterior to the base location, and rotated anticlockwise as in the $6^{\text {th }}$ position; 9) $10 \mathrm{~mm}$ posterior to the base location, and rotated clockwise as in the $7^{\text {th }}$ position; and 10) base location. 


\subsubsection{Head scanner}

The dummy was scanned 10 times. The following locations were used: 1 ) base location (center of the scanning volume of a head scanner); 2) $10 \mathrm{~mm}$ anterior to the base location; 3) $10 \mathrm{~mm}$ posterior to the base location; 4) $10 \mathrm{~mm}$ to the right of base location; 5) $10 \mathrm{~mm}$ to the left of base location; 6) rotated anti-clockwise (the placement procedure is specified in the protocol); 7) rotated clockwise (the placement procedure is specified in the protocol); 8) $10 \mathrm{~mm}$ anterior to the base location, and rotated anticlockwise as in the $6^{\text {th }}$ position; 9) $10 \mathrm{~mm}$ posterior to the base location, and rotated clockwise as in the $7^{\text {th }}$ position; and 10) base location.

\subsubsection{Landmark coordinates}

Coordinates of landmarks are calculated from each scan using the system software, and were saved in tab-delimited text format. Methods for determining the locations of marker stickers and for labeling landmarks depend on scanner systems.

\section{Evaluation procedure}

All calculations were performed in the Digital Human Research Center, AIST, using the same procedure.

\subsection{The ball}

The procedure for calculating the quality parameters is as follows: 1 ) the measured data are imported to Geomagic studio (Geomagic Inc.), and data points that do not belong to the ball surface are eliminated manually. 2) Coordinates of the center of the best-fit sphere are calculated according to the least squares method, using software developed for this purpose. The distance from each data point to the center of the best-fit sphere was calculated, and the radius of the best-fit sphere is obtained as the mean of these distances. 3) The error of diameter measurement (PS) is calculated as the diameter of the best-fit sphere minus the true diameter given by CMM. 4) The following statistics of the distances from data points to the center of the best-fit sphere were calculated: $N$, mean, standard deviation (SD of radius), minimum, maximum, P1, P5, P50, P95, P99 values. 5) Error of spherical form measurement (PFS) is calculated as the maximum distance minus minimum distance.

\subsection{Landmark locations}

The repeatability is evaluated for the landmark locations, because it is impossible to give the true values to landmark locations using CMM due to the complicated shape of the dummy. Only those landmarks that can be measured in each of 10 scans are evaluated.

\subsubsection{Pairwise superimposition}

The procedure is as follows: 1) Landmark coordinates obtained from Scan \#1 are used as the base data. 2) For each of other scan data, landmark were superimposed on those of the base data by minimizing the sum of distances between corresponding landmarks of the base data and that scan using quasi-Newton method, a non-linear optimization method. 3) For each of comparison, for each landmark, the error is calculated as the distance between the corresponding landmark locations. 4) Using nine comparison data, the mean and standard deviation of errors are calculated. 5) For each landmark, the mean error is reported.

This procedure is repeated using each of scan \#2 to scan \#10 as the base data. Also, mean error was calculated using the comparison results from all possible pairs of different scans.

\subsubsection{Simultaneous superimposition}

Landmark locations obtained from the 10 scans were superimposed simultaneously by minimizing the sum of distances between corresponding landmarks from all scans and for all landmarks by using quasi-Newton method. After the superimposition, mean error was calculated for each landmark using errors obtained from all possible pairs of different scans.

\section{Results and Discussions}

Eleven whole body scanners from seven manufacturers, four head scanners from two manufacturers, three foot scanners from two manufacturers, and one hand-held scanner (used for head scanning) were used to evaluate the proposed protocol. The dummy was not used for one whole body scanner 
and head scanners from one manufacturer. The dummy was scanned only once by the hand held scanner. All scanner systems that scanned a dummy could export the measured data. One whole body system did not export the ball measurements due to software specification [10].

\subsection{Measurement of the ball}

The ball was measured five times at each of nine locations using three of whole body scanners. Error of diameter measurement (PS) was consistent among the five repeated measurements: the maximum of the range of five PS measurements was $0.21[\mathrm{~mm}]$. However, error of spherical form measurement (PSF) varied considerably among the five repeated measurements. The maximum of the range of five PSF values at the nine locations was $14.7[\mathrm{~mm}]$ for a whole body scanner, Scanner-1. Standard deviation of radius is less influenced by irregular values than the minimum or the maximum: the maximum of the range of SD values among the five repeated measurements was $0.24[\mathrm{~mm}]$ for Scanner-1.

In some scanner systems, the measured area of the ball surface was small (Figure 5). Figure 5 shows that three whole body scanners, Scanner-4, Scanner-5, and Scanner-6, had smaller measured area of the ball than other three scanners. A small measured area can be due to the surface treatment of the ball was not proper for the system. We examined the measured areas of the dummy or human body surface for Scanner-4 and Scanner-5, and found that the measured area of the dummy was also small in these scanners. Figure 6 shows examples of measured area of the dummy.

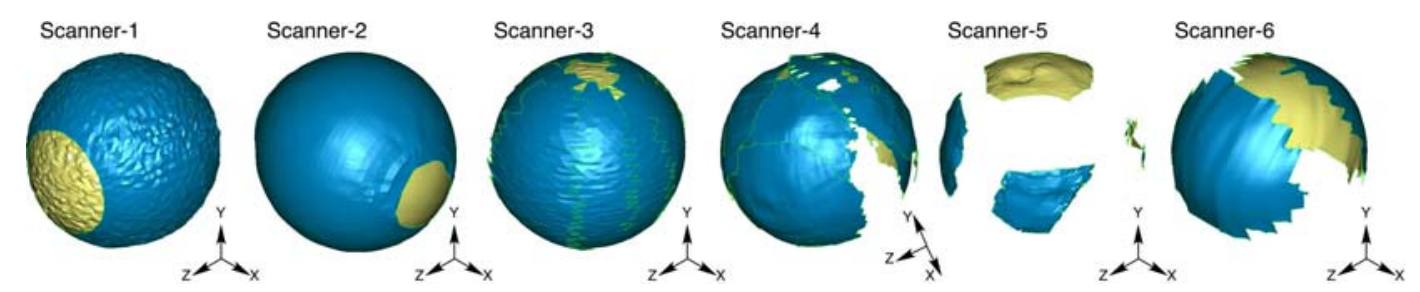

Fig.5. Examples of measured surface of the ball at location \# 3 by different whole body scanner systems.
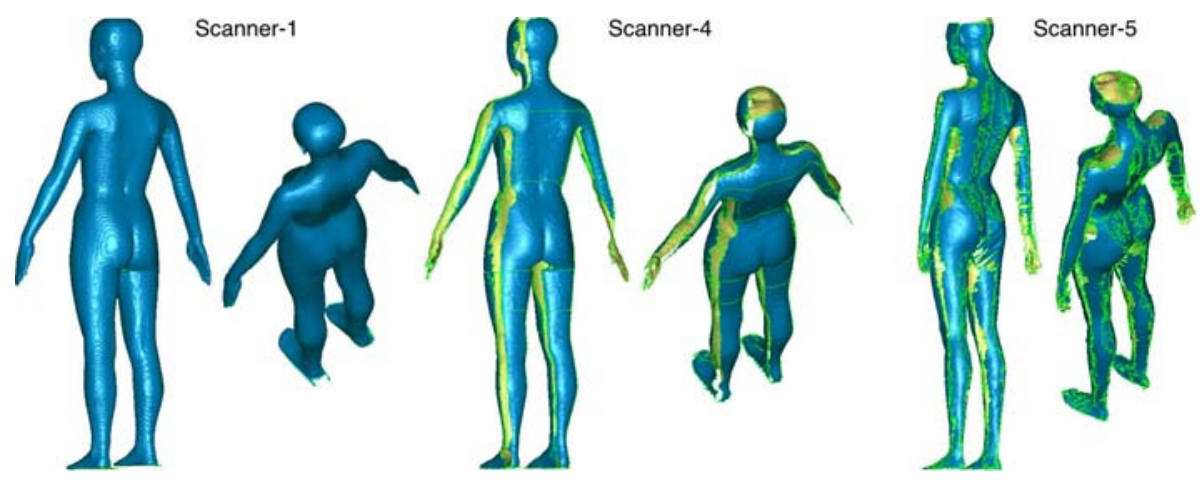

Fig.6. Examples of measured surface of the dummy by three different whole body scanner systems.

Figure 7 shows PS values at nine locations of three whole body scanners of the same model (Scanner-1). Different scanners had different results even if the model was the same. This suggests the effects of calibration on PS values.

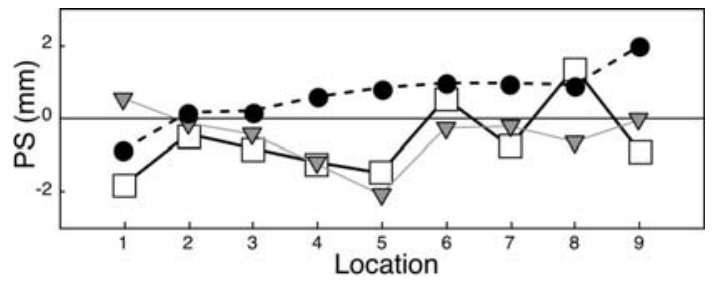

Fig. 7. PS values (error of diameter measurement) of three whole body scanners of the same system (Scanner-1). 
Figure 8 shows PS values at different locations of six different whole body scanners. PS values of the same scanner at different locations are sometimes considerably different. Figure 8 also shows that some scanners have smaller PS values and others have larger PS values. For example, PS values of Scanner-5 are much smaller than those of other scanners. Scan of the dummy by Scanner- 5 looks more slender than those by other scanners with larger PS values as shown in Figure 6.

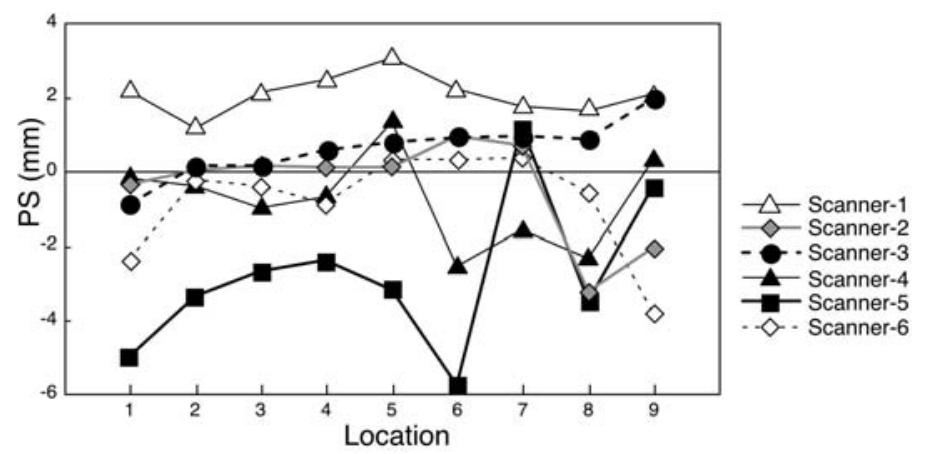

Fig. 8. PS values (error of diameter measurement) of six different whole body scanner systems.

\subsection{Landmark locations}

Figure 9 shows examples of evaluation results of landmark locations obtained by two whole body scanners, Scanner-1 and Scanner-3. When landmark coordinates obtained from scan \#1 to scan \#10 were used as the base data, the mean error can differ widely. Grand mean calculated using $10 \times 9=90$ comparison results is very close to the grand mean obtained by simultaneous superimposition in which results from all scans are superimposed simultaneously. Figure 10 shows examples of comparison between grand mean obtained by pairwise superimposition and grand mean obtained by simultaneous superimposition for three whole body scanners. Results of the two methods are very similar. Scanner-1 has the smallest mean error of landmark locations, though it had the largest error of diameter measurement among the three scanners (Fig. 8). Since the errors of landmark locations are influenced by performance of software for detecting and calculating landmark locations, scanners with smaller PS or PFS values do not necessary have higher repeatability of landmarks.
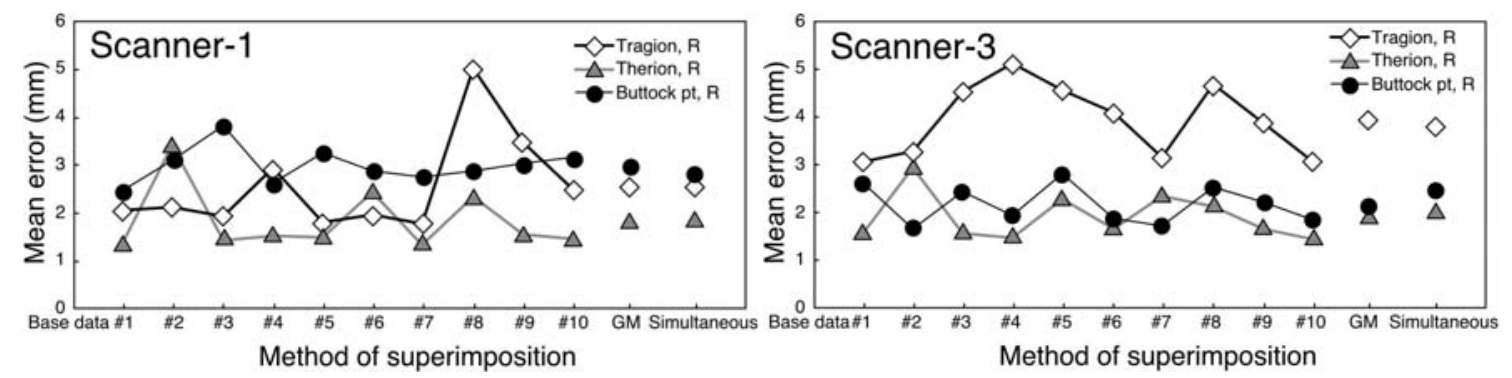

Fig. 9. Example of mean errors in landmarks when different scan is used as the base data or different method is used for superimposition. GM: grand mean.
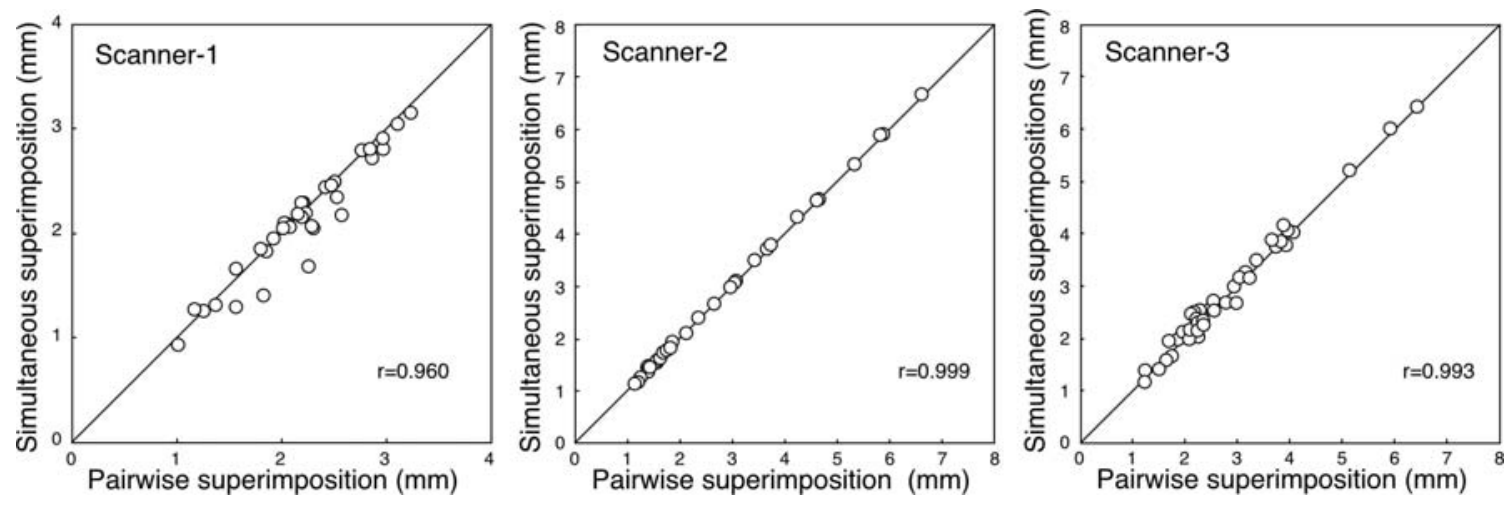

Fig.10. Comparison of mean error of landmark location between grand mean obtained by pair wise superimposition and grand mean obtained by simultaneous superimposition. 


\section{Discussion}

The protocol proposed in this study is intended to establish agreement between anthropometric database providers and data users. Database providers are expected to validate the accuracy of the scanner system they use by conducting the evaluation, and to assure users of the quality of scan-derived measurements. If the worst-case quality parameter of a scanner is better than the accuracy of traditional tools $(1 \mathrm{~mm})$, the database provider will easily convince data users that the data are accurate. Three of head scanners and all foot scanners satisfied this condition, while none of the whole body scanners used in the present study did.

Required accuracy may depend on the application of scan-derived measurements. Accuracy of measurements described by PS values and PFS values is usually not uniform within the scanning volume. Therefore it is important to examine results from all locations to evaluate the quality of landmark locations and surface shape especially for whole body scanners.

Measured area of the ball was small in some whole body scanners. In these scanners, the measured area of the dummy was also small. Since the size of occluded area influences the quality of surface shape and accuracy of landmark locations, it is an important aspect of the quality of scan-derived measurement.

\section{Conclusions}

We proposed a protocol for evaluating the accuracy of 3D body scanners using two types of test objects. The protocol was examined through a round-robin test. Proposed test objects were available for all except one whole body scanner, which did not export measurement of the ball. Error of diameter measurement and standard deviation of the distances between the center of the best-fit sphere and measured data points of the ball are useful for quantifying and comparing the accuracy of scan-derived surface shape. Mean error of landmark locations after superimposing repeated scans is useful for quantifying the repeatability of landmark locations. These parameters can provide a basis for the agreement between anthropometric data providers and data users. Since required accuracy depends on applications, it may be better to examine results from different scan locations to evaluate the quality of scan-derived measurements rather than considering only the worst case.

\section{References}

1. ISO 20685: 2005 3-D scanning methodologies for internationally compatible anthropometric databases.

2. Mochimaru, M. and Kouchi, M., (2000) "Statistics for 3D human body forms", SAE Technical Paper 2000-01-2149.

3. Allen, B., Curless, B. and Popovic, Z., (2003): "The space of human body shapes: reconstruction and parameterization from range scans", ACM Transactions on Graphics (ACM SIGGRAPH 2003), 22 : $587-594$.

4. JIS B 7441: 2009 Acceptance and reverification tests for coordinate measuring machines (CMM) with non-contacting probing systems.

5. VDI/VDE 2634 Part 1: 2002 Optical 3-D measuring systems Imaging systems with point-by-point probing.

6. VDI/VDE 2634 Part 2: 2002 Optical 3-D measuring systems Optical systems based on area scanning.

7. VDI/VDE 2634 part 3: 2008 Optical 3D-measuring systems - Multiple view systems based on area scanning.

8. Kouchi, M., Mochimaru, M., Bradtmiller, B., Daanen, H., Li, P., Nacher, B., and Nam, Y., (2012) A protocol for evaluatin the accuracy of 3D body scanners. Work: A Journal of Prevention, Assessment and Rehabilitation, 41 (Suppl.): 4010-4017.

9. Sato, O., Osawa, S., Takatsuji, T., Murakami, M., and Harada, R., (2008) Test artifacts for the verification of optical digitizers. Key Engineering Materials, Vols. 381-382: 553-556

10. Personal communication with the scanner maker. 